\title{
Sjogren - Larsson Syndrome
}

\section{Lt Col AK Dubey ${ }^{*}$, Lt Col RK Gupta ${ }^{+}$, Col Arvind Gupta ${ }^{\#}$}

MJAFI 2002; 58 : 352-354

Key Words : Ichthyosis; Mental retardation; Spastic diplegia

Qjogren - Larsson Syndrome (SLS) is a rare Neuroectodermal genodermatosis inherited as an autosomal recessive disorder. It seems more common in North Sweden than elsewhere in Europe and has been estimated as having the incidence of 10.2 in 1000,000 [1]. The disorder is characterized by the presence of congenital ichthyosis, mental retardation, and spastic di/tetraplegia [2]. We report a typical case of SLS and highlight latest aspects on the subject as regards imaging, gene mapping and enzymatic studies.

\section{Case Report}

6 year old male child, first of the three siblings was delivered preterm, spontaneously through vaginal route. There was no history of consanguinity, exposure to teratogens and birth asphyxia. Child was noticed to have dry scaly skin at the time of birth itself, and was diagnosed as a case of ichthyosis. He presented to us with dry keratotic,

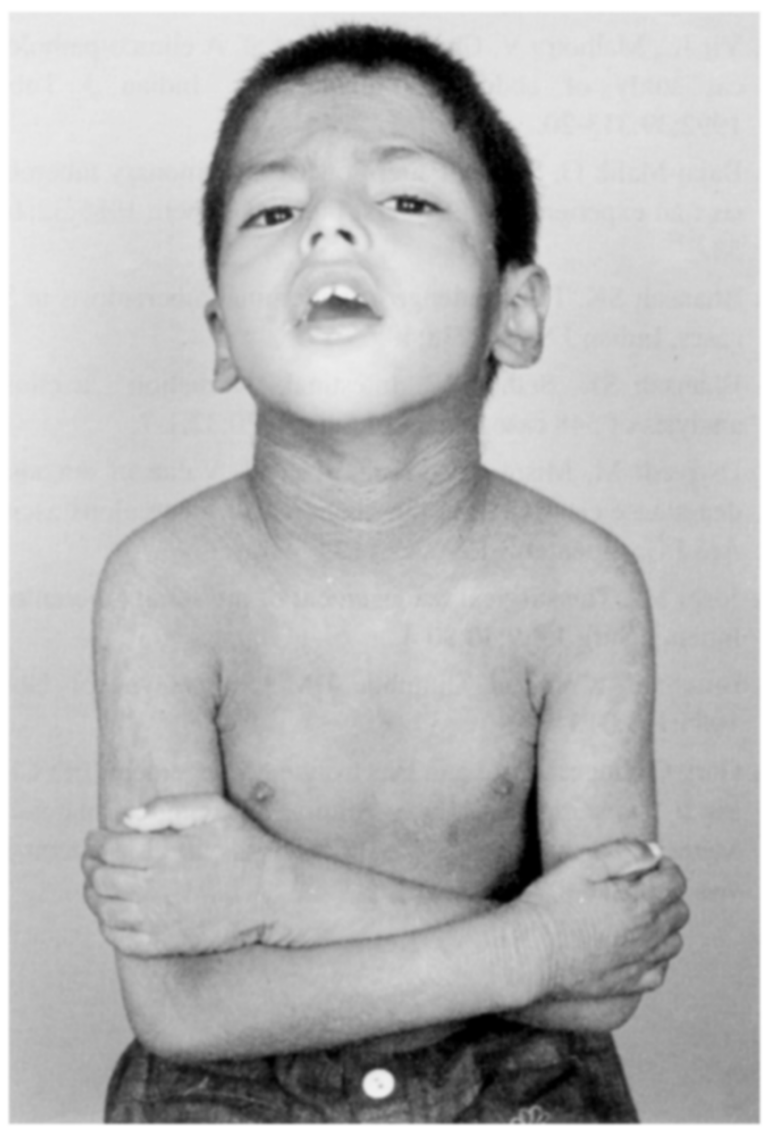

Fig. 1: Ichthyotic lesions on the body pruritic scaly skin lesions, delayed motor and intellectual milestones. These disabilities were first detected during late infantile period. He could speak only in short sentences. There was no history of seizures. Examination revealedchild weighing $16 \mathrm{Kg}$, head circumference $-49 \mathrm{~cm}$, vital signs within normal limits. There were generalized dry scaly ichthyotic lesions all over the body, predominantly over the flexor aspect of neck, axilla and lower abdomen. Neurologically he had mild retardation of higher mental functions, IQ being 55 on DSIS scale. There was no cranial nerve deficit. Spasticity of all the four limbs, lower limbs being more severely affected than upper was present. He had scissoring gait and contractures of the tendoachilli bilaterally. Muscle power was grade III-IV in all four limbs. All deep tendon jerks were exaggerated and plantars were extensors. There were no sensory deficits or cerebellar signs. Ocular fundi showed glistening white spots surrounding the macular region.

Investigation profile revealed normal blood count. Electro encephalogram (EEG) did not show epileptiform discharges. Thyroid hormone profile was within normal limits. Both computerised tomography (CT) scan and magnetic

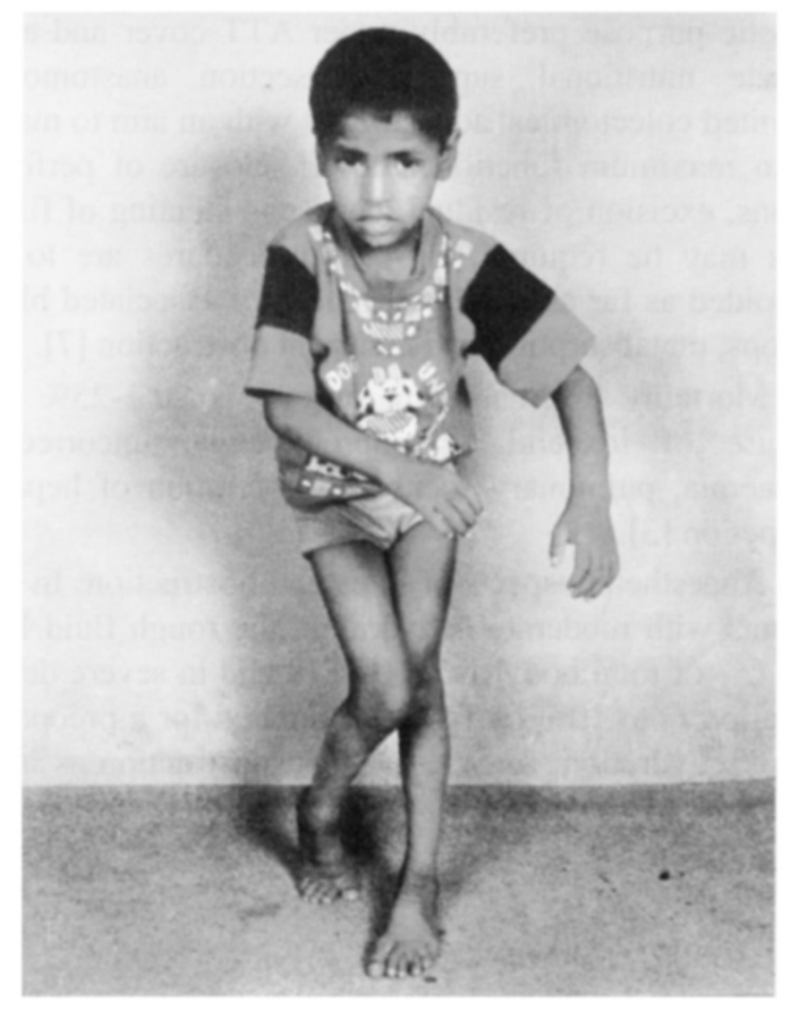

Fig. 2: Spastic tetraplegia

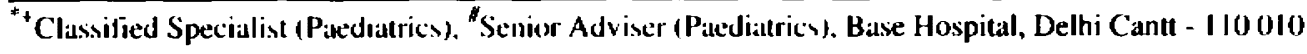




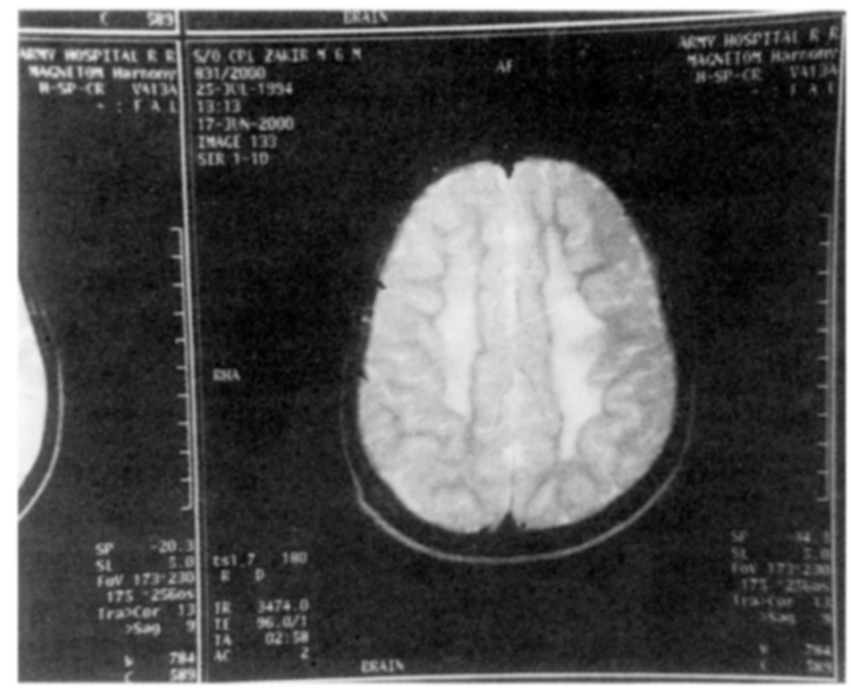

Fig. 3: MRI cranium showing white matter degeneration involving periventricular, centrum semiovale, corpus callasum and parieto-occipital region

resonance imaging (MRl) were suggestive of white matter degenerative disorder involving periventricular, centrum semiovale, corpus callosum and parieto-occipital regions. Skin biopsy showed marked hyperkeratosis of epidermis with mild perivascular infiltration of the lymphocytes. No vacuolation of the stratum spongiosum was seen. Gene mapping and enzymatic studies could not be carried out for want of facilities.

\section{Discussion}

Sjogren and Larrson characterized this syndrome for the first time in 1957 after studying 28 patients in 13 families in the North Swedish province of Vasterbatten [3].

The skin disorder becomes evident after the first few months of life with scaling and hyperkeratosis which in some cases may be quite severe [4]. Some affected individuals are born as collodian babies [5]. Pruritus is a characteristic feature [6]. The histological features of the skin include hyperkeratosis, papillomatosis, acanthosis and mildly thickened granular layer. Electron microscopy shows lamellar membranous inclusions in the granular and cornified cells. This fatty alcohol accumulation is thought to be responsible for the cutaneous symptoms [2].

Neurologic component usually starts between 4 and 30 months and remains static after puberty. There is usually spastic diplegia, occasionally tetraplegia with mental retardation, epilepsy, speech defect, dental, skeletal deformities and retinal changes have been reported [7]. Cognitive impairment is often profound but sporadic cases have normal intelligence. Seizures occur in approximately $40 \%$ of the cases. In some patients the only sign suggestive of neurological involvement is hyper-reflexia. Pseudo bulbar dysarthria and delayed speech are common. Mental retardation is usually of mild to moderate range and its severity coincides with degree of spasticity [8]. One third of the patients exhibit a distinctive, perhaps pathognomonic presence of glistening white dots surrounding the macular region of the retina, which first appear after several years of age. Visual acuity may be decreased and photophobia may be present. It remains to be determined whether loss of visual acuity is a consequence of the retinal deposit or dysmyelination in optic tract.

In contrast to central nervous system (CNS), peripheral function is spared in SLS. Interestingly, there is no correlation between neurologic symptoms with ichthyosis and ocular symptoms [8]. SLS is diagnosed by the characteristic triad as described above. This condition should be suspected in a child with congenital ichthyosis. The diagnosis is confirmed when signs of spasticity and mental retardation are present before the age of three years [8]. Perifoveal glistening spots are almost pathognomonic. Further confirmation of the diagnosis can be done by MRI studies which show retarded myelination or variable degree of dysmyelination. MR spectroscopic findings suggest an accumulation of long chain fatty alcohol intermediates resulting in retarded myelination and dysmyelination. $1 \mathbf{~ H}$ MRS of the periventricular lesions showed by MRI revealed high lipid and low $\mathrm{N}$ acetyl-aspartate peaks, which are considered pathognomonic of SLS.

Despite the myelin abnormality SLS patients do not show a progressive neurodegenerative course and most survive in to adulthood [6].

Rizzo et al (1988) reported that oxidation of long chain fatty alcohols was deficient in SLS patients due to a dysfunctional enzyme complex fatty alcohol : nicotinamide - adenine dinucleotide (NAD+) oxidoreductase (FAO). In this enzyme complex there are different proteins of which fatty alcohol dehydrogenase (FADH) catalyses the oxidation of fatty alcohol to fatty aldehyde dehydrogenase (FALDH) catalyses the oxidation of fatty aldehyde to fatty acids. Detailed biochemical studies have revealed that the primary enzymatic defect lies in the microsomal FALDH component. This deficiency can be demonstrated in cultured fibroblasts of parents and carriers. Prenatal diagnosis of SLS can be accomplished by enzymatic studies of amniocytes and cultured chorionic villi cells or on histological examination of fetal skin biopsy.

SLS patients have profound deficiency of FALDH. FALDHcDNA has been cloned and has been found to map to the SLS locus on chromosome 17p11.2. Sequence analysis of FALDH amplified from fibroblast mRNA and genomic DNA from three unrelated SLS patients reveal distinct mutations, including deletions, 
an insertion and a point mutation. The cloning of FALDH and identification of the mutations opens up the possibilities for developing therapeutic approaches to ameliorate the neurological and cutaneous symptoms of the disease.

\section{References}

1. Jagell S, Gustavson KH. Holmgren G. Sjogren-Larsson Syndrome in Sweden. A clinical, gentic and epidemiological study. Clin genet 1981:19:233-56.

2. Rizzo WB. Semin Dermatol 1993;12(3):210-8.

3. Sjogren T. Larsson T. Oligophrenia in combination with congenital ichthyosis and spastic disorders. Acta Psychiatr
Neurol Scand 1957;32(Suppl. 113):1-113.

4. Traupe H. The Ichthyosis. Berlin. Heidelberg:Springer-Verlag: 1989.

5. Bonafe JL, Peyraga C, Lafitte JM, et al. Le syndrome de Sjogren-LaRSSON. Ann Dermatol Venereol 1987;114:94755.

6. Rizzo WB. Sjogren-Larsson syndrome, explaining the skin brain connection. Neurology 1999;52:1307-8.

7. Selmanowitz VJ, Porter MJ. The Sjogren-Larsson syndrome. Am J Med 1967;42:412-22.

8. Jagell S, Heijbel J. Sjogren-Larsson syndrome:physical and neurological features. Helv Paediatr Acta 1982;37:519-30. 